\title{
Generando una comunidad de práctica en una red social. Análisis de un caso ${ }^{1}$
}

\section{Generating a practice community through a social network. A case analysis}

\author{
Joaquín PAREDES-LABRA, Agustín DE LA HERRÁN-GASCÓN y \\ Daniel VELÁZQUEZ-VÁZQUEZ \\ Universidad Autónoma de Madrid y Universidad Nacional Autónoma de México
}

Recibido: Febrero 2012

Aceptado: Abril 2012

\section{Resumen}

Este artículo explora la generación de una red de práctica en una red social para repensar la formación docente continua.

Se presenta un estudio de caso de una red en la que participan 70 profesores de todos los ámbitos de conocimiento en una universidad mexicana convencional y que han hecho innovaciones en sus cursos. Los docentes han modificado sus programas para incluir elementos de creatividad, se desarrolló un proyecto con los estudiantes, se crearon redes físicas y virtuales con sus compañeros. Se presentan los cambios, fortalezas y debilidades de la red social que cada docente utiliza como un medio para la práctica reflexiva.

Palabras clave: creatividad, redes sociales, pensamiento práctico del profesor, educación superior.

\begin{abstract}
This paper explores a practice community generation in a social network to rethink continuum teacher training.

We present a study that included 70 teachers from all domains of traditional Mexican universities who made innovations in their courses. Teachers modified their programs to include elements of creativity, they developed a project with students, created physical and virtual networks with peers. Here we present changes, strengths and weakness of the social network that each teacher used as a medium for reflective practice.
\end{abstract}

Keywords: creativity; teachers' practical thinking; social networks; Higher Education.

\footnotetext{
${ }^{1}$ Este proyecto ha sido financiado con una ayuda de la Agencia Española de Cooperación Internacional, proyecto A103554511.
} 
La participación de personas con propósitos comunes en redes sociales en Internet es una tendencia en todos los ámbitos de la vida en sociedad y la cultura contemporánea, tal y como muestra el notable crecimiento de redes sociales genéricas (Facebook, Twitter) o temáticas (alojadas por ejemplo en Ning).

Las redes temáticas, más desconocidas por haber dejado de ser gratuitas en su mayoría, están organizadas en torno a intereses específicos y para una audiencia determinada. Una persona o grupo deciden implementar una red temática a la que generalmente se pueden suscribir potenciales usuarios mediante invitación (podría ser pública, aunque es desaconsejable por la especificidad de la temática) y donde van a encontrar a otros usuarios con los que comparten objetivos y temas. El funcionamiento de la red temática, una vez registrado, es similar al resto de redes -lo que en origen era potestativo de estas redes temáticas-: permite el posteo de información textual e imágenes, el mantenimiento de un blog personal, el empotrado de documentos y vídeos, la participación en foros promovidos por él o por otros, la generación de grupos dentro de la propia red, el anuncio de eventos y otras funcionalidades que pueden ser implementadas por el administrador de la red con la intención de organizar la interacción rica entre sus participantes y el logro de los objetivos que se propone. Tuvieron enorme importancia en educación para organizar cursos por el método de proyectos hasta que estas redes se hicieron de pago.

En este trabajo se describe la generación de una comunidad de práctica de docentes universitarios en una red social del tipo Ning, a partir de un caso. En el caso se describe el proceso de participación de quienes se integran en la red, sus dificultades y sus posibilidades para el desarrollo profesional.

\section{Marco teórico}

Entendemos como comunidad de práctica a un colectivo de docentes que comparte una visión de la enseñanza y eligen el debate continuado y público de ideas como un vehículo para mejorar su práctica y desarrollarse profesionalmente (Wenger, 1998; Lieberman y Miller, 2001).

Estas comunidades comparten sus incertidumbres y experiencias, buscan marcos explicativos para su práctica, proponen cursos de acción y los evalúan. Se abre la posibilidad de generar conocimiento profesionalmente valioso a partir de la escritura pública y el debate con los colegas.

Hablamos, más allá de los modelos de cursillo o de formación en cascada, de propuestas de autoformación. Lógicamente, hay otras propuestas dentro de estas comunidades que las convierten en espacios más complejos, y que han sido analizadas en otro lugar (Paredes, 2011; Feldman y Weiss, 2010; James, 2009): procesos de investigación-acción emprendidos, aprendizaje colaborativo en red generado, indagación profesional realizada, emergencia de nuevos usos profesionales de las redes como entornos personales de aprendizaje percibidos, cambio de los supuestos de enseñanza y aprendizaje en educación superior. 
La comunidad que se procuró constituir surge a partir de un proyecto de la Agencia Española de Cooperación Internacional (AECID) sobre la mejora de la enseñanza en la universidad al incorporar la creatividad y las TIC. Nuestro contexto de enseñanza se compone de prácticas docentes universitarias tradicionales, formales, centradas en el profesor, en universidades de México. Participan profesores universitarios en todos los ámbitos. Nuestro objetivo es promover una enseñanza más abierta a través de la creatividad (como característica del conocimiento) (Torre, 2006; Herrán, 2008a, 2008b) y las TIC para un amplio grupo de profesores a los que inicialmente les interesa mucho esta forma de trabajar.

Las redes sociales ya acogen colectivos docentes diversos. Didáctica Universitaria, Dioe, Internet en el aula y Red docente de Tecnología Educativa son algunos ejemplos que agrupan a varios miles de docentes sobre las temáticas que sus nombres indican.

Una red social puede hacer muy poco sin un esfuerzo por dinamizar y promover la participación, que es responsabilidad de todos. Las comunidades profesionales en el campo de la enseñanza han sido estudiadas en cuanto a sus dinámicas y su éxito. Como Stuckey y Barab (2007) señalan, si algo caracteriza estos espacios son las tensiones entre:

- Trasmitir contenido o promover participación.

- Apertura o encauzamiento de la participación.

- Estructura predefinida o emergente.

- Facilidad de uso o sociabilidad.

La comunidad es útil para el crecimiento profesional. Está concebida como un vehículo y algunos de los participantes junto con sus promotores están animando a la participación. No hay una propuesta preconcebida de mejora, sólo una idea de mejorar el diagnóstico de las prácticas y ampliar el protagonismo de los estudiantes en la docencia que practican estos docentes.

En todas las comunidades tiene cierta importancia que ocurran encuentros cara a cara como un factor explicativo de la posterior participación virtual en la comunidad.

\section{El caso: "formacionmediantecreatividad", una red social para la mejora de la enseñanza}

"Formacionmediantecreatividad" es una comunidad al servicio del desarrollo profesional. Su temática es la aplicación de la creatividad y las TIC para mejorar la Educación Superior, a partir de un análisis de la enseñanza practicada y de cursos de acción definidos por los docentes que son nuevamente analizados. En 2010 adoptó algunos procedimientos específicos para nutrir el colectivo e incentivar la participación, tales como un curso presencial previo abierto a cualquier participación, centrado en técnicas sobre creatividad y menos sobre TIC, un plan de entregas posterior en la red y unos facilitadores con una visibilidad mínima. Al analizar el caso que se presenta, se genera- 
ron nuevas directrices para el proceso de incorporación de participantes, el tipo de formación previa, el papel de los facilitadores y la forma de incentivar la participación.

Había 70 participantes inscritos. Mediante las preguntas descriptivas de un cuestionario sobre creatividad y TIC en la enseñanza practicada que se aplicó antes de comenzar el proceso formativo (Paredes y Herrán, 2011), se supo que la mayoría de los participantes eran mujeres, con edades mayoritariamente comprendidas entre 30 y 40 años, y que provenían en su mayoría (72\%) de uno de los 5 campus de la UNAM alrededor del Distrito Federal. La mayoría eran profesores de Ciencias Sociales, en particular los estudios de Educación (gráfico 1).

Gráfico 1. Características de los participantes. Grado en el que enseñan

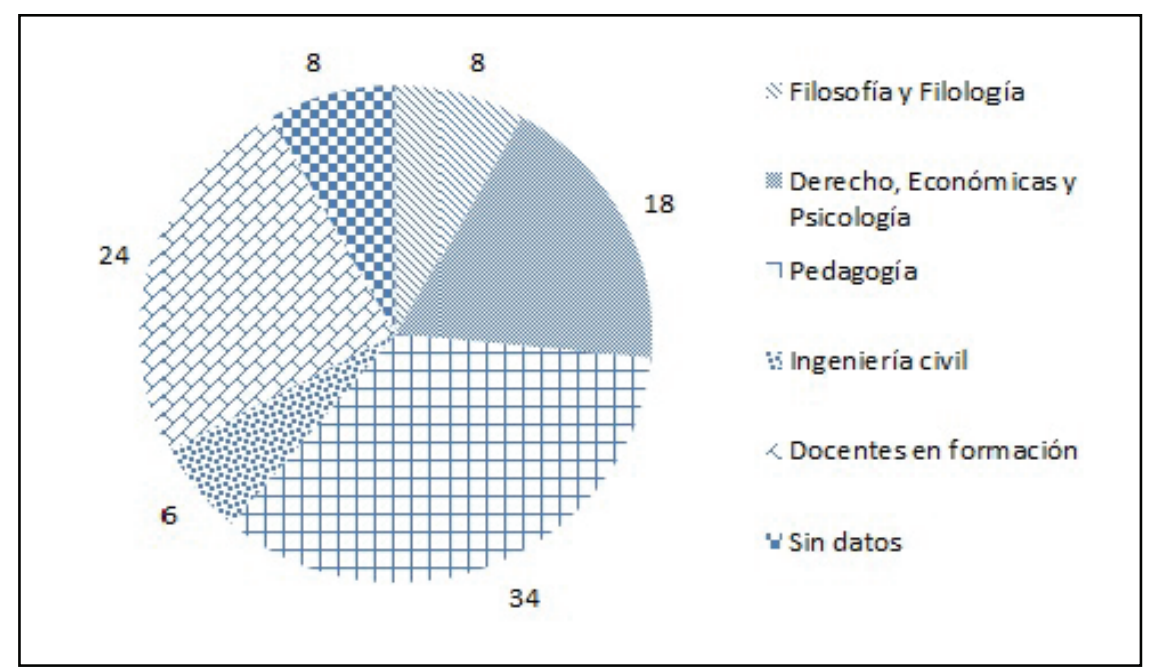

Un porcentaje significativo de los docentes no habían recibido formación pedagógica para aplicar en su enseñanza (48\%). Por lo general, no tenían una amplia experiencia docente (casi el 70\% tenían experiencia de menos de 10 años como profesor universitario) (gráfico 2). 
Gráfico 2. Características de los participantes: experiencia docente

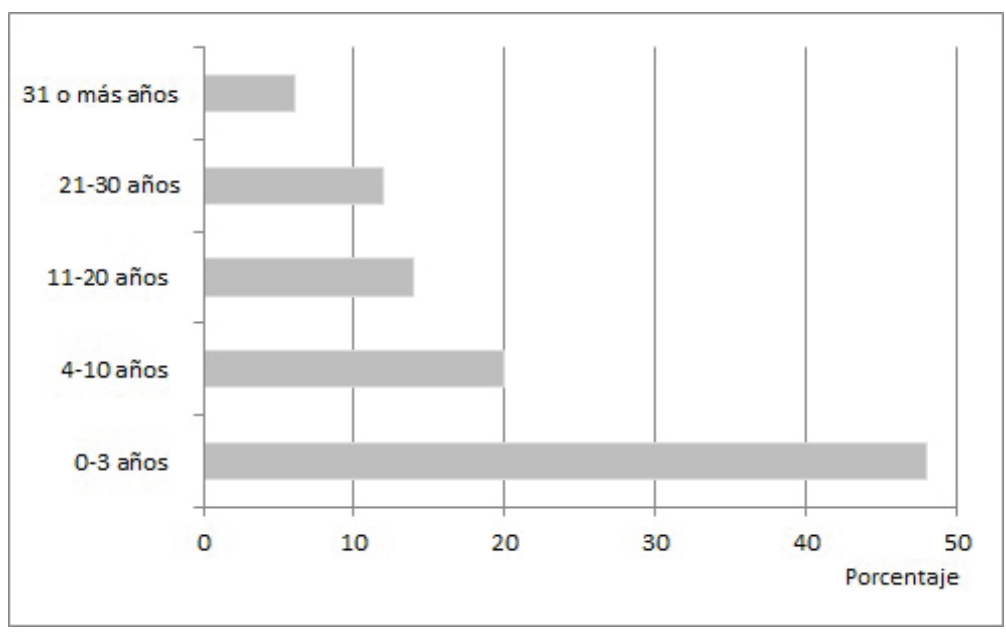

Otras características de la muestra se resumen en:

- Ninguno de los participantes se veía como poco creativo.

- Casi todos los encuestados consideran fundamental la competencia creativa para el desempeño profesional de los graduados.

- Cuatro quintas partes pensaba que había ganado en la creatividad en los últimos años.

- Tres cuartas partes de los profesores afirmaron promover procesos educativos innovadores.

- Cuatro quintas partes informaron de que su enseñanza se orienta más hacia las habilidades cognitivas (observar, sintetizar, relacionar, inferir) que el aprendizaje de los contenidos.

- La mayoría consideraba a sus estudiantes como capaces de participar en una enseñanza que sacara de ellos respuestas creativas.

- Todos dijeron que el aprendizaje activo que se ocupa de la creatividad ayuda a los estudiantes a avanzar en su formación. Compartir la responsabilidad con los estudiantes es relevante para el aprendizaje formativo.

- Creen en el potencial de cada grupo y cada alumno para mejorar, que la cordialidad y la buena comunicación con los estudiantes es muy importante para el desarrollo de la enseñanza creativa. Todas se benefician de las intervenciones creativas de sus alumnos para enriquecer sus clases.

- Había una percepción general de libertad de organizar su enseñanza de manera activa. 
Para caracterizar la red social como caso seguimos las recomendaciones de Yin (2009) quien reclama para los estudios de caso la presencia de un protocolo, unas preguntas directrices, un plan de recogida de información y un informe con alguna lógica, al menos una descriptiva de lo que allí sucede. La elaboración de un informe cronológico de lo que allí aconteció, sugiere Yin, puede ofrecer también explicaciones de por qué sucedió, con los procedimientos de triangulación habituales en la etnografía, cuyas técnicas y forma de análisis se adoptan en este estudio. En este caso, las evidencias las aportan los profesores en la red, mediante comentarios y emails que generan, así como fotografías y vídeos de los estudiantes que son diferentes evidencias de diversos agentes participantes, junto a las interacciones que se producen con los facilitadores.

La red social nace impulsada por un proceso formativo en el que los promotores o facilitadores quieren ser poco visibles. Se desea recoger información relevante de todo lo que ocurra dentro de la red para poder comprender su viabilidad e ilustrar a futuras redes.

El temor de los promotores, investigadores y facilitadores de la misma, es el mismo que el que rodea toda la observación participante, contaminar lo que allí deba ocurrir y que es objeto de conocimiento. Sus intervenciones se producirán sólo cuando la red esté sin actividad y cuando lo reclamen los participantes, que será en alguna ocasión.

El trabajo de campo previsto, por tanto y como se ha anunciado, tiene una vocación etnográfica. Sin embargo, los investigadores tienen experiencia en redes más numerosas y son conscientes de que una comunidad pequeña (que no alcanza el centenar de profesores) quizá no tenga la misma dinámica (participación, interacciones, que serán evidencias para analizar) que las redes de un millar largo de profesores que han frecuentado antes.

Este proyecto pretendía que los docentes participantes buscaran respuestas a varias preguntas: ¿Están los estudiantes preparados para responder de manera abierta y creativa a los problemas que les plantean en la enseñanza? ¿Los proyectos propuestos a los estudiantes les permitan responder de manera creativa? ¿Qué tipo de relaciones profundas fomenta la investigación y las respuestas más creativas de los estudiantes en la clase? ¿Qué papel juegan en recursos de respuestas creativas?

Los investigadores querían entender el fenómeno que se iba a producir en el contexto de la red social.

El plan original de recogida de información de esta comunidad, que nos permitiría analizar su funcionamiento y extraer algunas consecuencias para el futuro, tenía como pauta un proceso de investigación acción (figura 1). Se generarían algunos documentos, se abrirían momentos de debate (que podrían quedar registrados en la red social), y se tomarían decisiones y cursos de acción formalizables en nuevos documentos, interacciones y evidencias. 


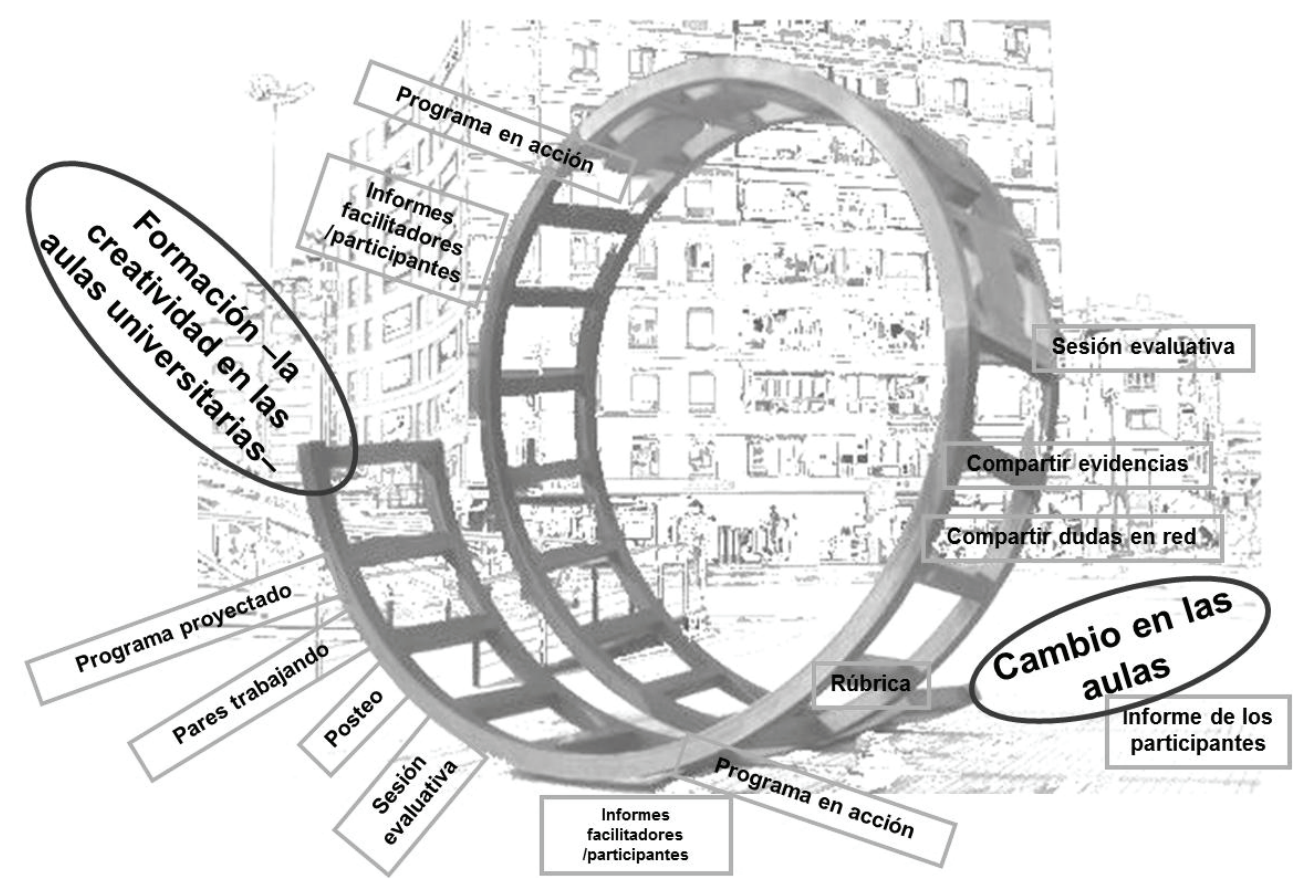

Figura 1. Proyecto Formacionmediantecreatividad

Tras un proceso formativo que había ocurrido antes del comienzo del semestre, al que se sumaron voluntariamente un amplio colectivo de participantes arriba descrito, cada profesor participante compartió en la red social un nuevo programa para alguna de las materias que imparte. Estaba obligado a incluir una o más de las técnicas creativas abordadas en el proceso formativo. Nos preocupaba, como se manifestó al grupo en repetidas ocasiones, más que la formalidad de la inclusión de una técnica de creatividad en un programa, la apertura de una reflexión sobre la propuesta de práctica docente y la posible interacción y crecimiento al compartir otras propuestas. Interesaba más el encuentro que se estaba produciendo para transformar la práctica.

El proceso se retrasaba. Cuatro meses después del establecimiento de la red social, el proceso fue reactivado por un mensaje de los facilitadores a los profesores a continuar su formación a través de la web, como se convino en la realización del curso, y les recordó que iban a recibir la certificación después de haber completado el curso virtual. Estos recordatorios se repitieron varias veces durante los meses siguientes, cada vez con una mejor respuesta de los que había permanecido en silencio.

Veinte profesores se unieron en grupos de manera espontánea sobre la base de las técnicas de enseñanza (algunos pertenecían a más de una): mapas conceptuales ( 8 profesores), autobiografías, reconstrucciones e invenciones (5), trabajo con multimedia (5), proyectos didácticos (5), fotos, imágenes y leyendas (4), uso emotivo de textos (4) y estudio de caso (3). De las 8 técnicas elegidas, 6 estaban basadas en las TIC. 
Tras esta propuesta, hubo un informe de progreso, preparado por los facilitadores y puesto a disposición del grupo para generar un debate. En ese informe, distribuido por correo electrónico, se observa que, en general, los programas eran muy pobres. No tenían metodología variada. Hubo una formulación débil de propósitos. El contenido era sólo conceptual. Los programas no se refirieron a la diversidad de las competencias de los estudiantes. No hubo diversidad de técnicas de evaluación. Hubo poca flexibilidad en la aplicación de técnicas de creatividad. En el informe se animaba a una mejora de la planificación y desarrollo ricos en el aula. No hubo comentarios.

El siguiente momento era poner en práctica el plan. Esto continuó hasta el final del semestre siguiente, e involucró varios meses de trabajo en donde se recolectaron pruebas en relación con lo que sucede en las aulas con el fin de comprender mejor el método de enseñanza aplicado.

Se animó a que las propuestas formuladas se debatieran con compañeros y colegas, de tal forma que aparecieran equipos locales de apoyo a las propuestas. Generalmente, cada equipo local se reunió dos veces por semana para discutir los problemas que surgieron, y las soluciones y las dudas se publicaron en la red. Durante las reuniones, las discusiones se llevaron a cabo en relación con las técnicas, lo que podría lograrse y su eficacia con el grupo de estudiantes.

Al mismo tiempo, se recibieron mensajes en la red social acerca de la marcha de los programas que constituyen las entradas personales de cada docente. Hubo también fotos y videos de las clases, y referencias de otros lugares de Internet donde se estaban posteando producciones de los estudiantes- Esta dinámica proporcionaba un diálogo fructífero, en la medida que ejemplificaba cursos de cambio que podían ser adoptados por los demás docentes. Hacen que sea posible establecer un diálogo sobre el avance de los proyectos.

$\mathrm{Al}$ acabar el semestre, se generó un informe de resultados, a partir del análisis de las evidencias presentadas por 26 participantes en la red social, con 12 profesores que ofrecen nuevos programas y el resto con diferentes problemas, sobre todo de tiempo para llevar sus programas a la práctica. En los programas, como principal técnica de creatividad aparecían 3 ensayos, una simulación, 2 estudios de caso, una propuesta multimedia, 3 mapas mentales y 3 proyectos. Siete profesores proporcionaron evidencias de las actividades de sus grupos: 2 fotos, 1 mapa, 1 informe, 1 vídeo, 2 sitios web (tabla 1). 


\begin{tabular}{|c|c|c|}
\hline Caso & $\begin{array}{l}\text { Principal actividad que introdu- } \\
\text { ce creatividad }\end{array}$ & Evidencias \\
\hline 1. & Mapas mentales & $\begin{array}{l}\text { - } \quad \text { Manifiesta dificultades: la red social no me } \\
\text { ofreció ayuda cuando la pedí. Pasó mucho } \\
\text { tiempo y nadie contestaba. }\end{array}$ \\
\hline 2. & Performance de los estudiantes & $\begin{array}{l}\text { - Al introducir la técnica, mi grupo de } \\
\text { estudiantes pensó que era un juego y me } \\
\text { desanimó a continuar trabajando }\end{array}$ \\
\hline 3. & Ensayos & - \\
\hline 4. & Ensayos & - \\
\hline 5. & Ensayos & - \\
\hline 6. & Simulación & - $\quad$ Entrevistas en video y fotos del trabajo \\
\hline 7. & $\begin{array}{l}\text { Estudio de caso (como técnica } \\
\text { docente) }\end{array}$ & - $\quad$ Fotos \\
\hline 8. & $\begin{array}{l}\text { Estudio de caso (como técnica } \\
\text { docente) }\end{array}$ & - Vídeo \\
\hline 9. & Multimedia & Posteo \\
\hline 10. & Mapas mentales & Posteo \\
\hline 11. & Mezcla de métodos & Web Site \\
\hline 12. & Proyecto & Web Site \\
\hline
\end{tabular}

Tabla 1. Docentes que completan el proceso, actividad principal y evidencias aportadas

La red social proporciona un medio para intercambiar y comentar el trabajo del curso, qué y cómo se enseña. Da respuestas a dudas de colegas y permite establecer nuevos cursos de acción. Además, aunque con una dinámica novel-experto, durante el semestre los participantes se refieren a todo tipo de dificultades, esperanzas y miedos. Los datos obtenidos permitieron al grupo crecer en el conocimiento de la creatividad aplicada a su propia enseñanza y la naturaleza misma de su propia enseñanza. Una comunidad de práctica se institucionalizó.

Las dificultades de participar nos hablan de la dificultad de la colaboración en dinámicas donde no existen estructuras previas, de promover la participación entre aquellos a quienes nunca se les ha pedido que lo hagan, y de transformar la educación a través de la creatividad, donde no hay tradición de dar voz a los estudiantes.

Mediante correos electrónicos, los participantes expresaron disculpas por la demora en la interacción en la red, la incertidumbre sobre una hipotética respuesta correcta esperada por los facilitadores, y el deseo de avanzar en el compromiso asumido en la formación previa. Con el fin de reactivar el proceso, el equipo de investigación pasó algún tiempo reflexionando sobre dinamizar la interacción. Los investigadores querían evitar la simple recepción de una programación. Sin embargo, también se temía que los esfuerzos pedidos al grupo podrían ser demasiados. El equipo decidió hacer lo siguiente:

1. Recibe todo el material generado por cada participante por correo electrónico, y solicita permiso para publicarlo simultáneamente en la red.

2. Anuncia la presencia de una técnica en la red sólo después de que un porcentaje significativo de los profesores han contribuido. 
3. Da información al grupo y construyen juntos el conocimiento mediante la reelaboración.

4. Visualiza este diálogo en una lista de correo, foro, chat.

5. Solicita alguna evidencia de los resultados de los estudiantes.

Hubo algunos intercambios asíncronos sobre estos pasos, y todos parecían satisfechos y con ganas de hacer algo más.

Algunos docentes plantearon cuestiones trascendentes: "¿Cómo yo como docente incorporo nuevas técnicas a fin de fomentar progresivamente el desarrollo de lo que podría constituir una estrategia de trabajo nuevo?".

Hemos recibido evidencias con las fotografías de los estudiantes que trabajan juntos, y vídeos donde los estudiantes realizan testimonios de lo que ocurrió en las prácticas transformadas.

Al final del semestre se pidió a los participantes, a la luz de lo que sus compañeros presentaban, que hablaran sobre el futuro de sus proyectos. Una serie de respuestas se formularon:

- Los docentes estaban interpretando y asumiendo respuestas creativas de sus alumnos.

- Los estudiantes parecían dispuestos a dar respuestas creativas.

- Los estudiantes apreciaban que sus profesores hubieran cambiado el tipo de participación en sus aulas.

- Algunos recursos utilizados se asociaron a los cambios adoptados.

Otras preguntas quedaron sin respuesta. Particularmente una muy importante, que habla de la generación de la comunidad de práctica: ¿El diálogo en las redes físicas y sociales ayudaron a mejorar mi comprensión de cómo hacer otro tipo de enseñanza es posible?

La red sigue trabajando, e incorporará estas propuestas.

En resumen, uno de cada diez profesores que originalmente se unió al proyecto ha decidido llevar a cabo un proceso de cambio hacia la formación mediada por la creatividad. Se trata de una proporción considerable en comparación con nuestras experiencias con foros generados (que se considera exitoso cuando se obtiene una aceptación de un 1\%), y en comparación con grupos similares que participan de vez en cuando (como observadores) en la vida de redes sociales, y que no superan el 5\% de participación.

\section{Conclusiones}

En 2011 se adoptaron algunos procedimientos específicos nuevos para mantener la red social en marcha como espacio para el desarrollo profesional de docentes universitarios. Como se indicó, el caso que se presenta generó nuevas directrices para el proceso de incorporación de participantes (entre colectivos que tenían un deseo de cambio 
que ya estaban al menos informalmente organizados, o bien que tenían responsabilidades de coordinación), el tipo de formación previa (con una variedad temática que incorporó de forma expresa técnicas de diagnóstico de la realidad, tutela para estudiantes como estrategia de apertura a una enseñanza centrada en ellos, y más TIC con un componente procesual y emotivo más que demostrativo), el papel de los facilitadores (menos instrucciones de participación) y la forma de incentivar la participación (certificar cuando se acabara todo el proceso).

Todo ello porque los resultados son modestos, particularmente en las interacciones dentro del grupo, que son el sentido de una comunidad (más que la reacción a los comentarios de los facilitadores).

La creatividad y las TIC son reclamos muy poderosos para transformar la práctica. Sin embargo, no parece lo suficientemente atractivo como para provocar una avalancha de participación. Fue invadida por otros factores condicionantes.

Nos pareció que el compromiso de los participantes con la comunidad no pudo ser muy alto. Las condiciones de trabajo de los profesores universitarios en México (aunque no se declararon segundas ocupaciones), el soporte emocional (que se confiaba a grupos locales autoconstituidos) y la libertad para participar una vez se había obtenido certificación ya eran suficientemente poderosos obstáculos y eximentes. El tamaño y estructura de la red y la forma de promover la participación también pueden haber sido obstáculos. Las investigaciones de Stuckey y Barab (2007), aunque no son concluyentes al respecto en estos aspectos, no las recogen. Nuestro caso nos hace inclinarnos a pensar en las primeras (proletarización, emotividad, certificación) como las razones más poderosas para las dificultades de funcionamiento de esta red.

Entre los cambios observados, se puede decir que:

- La educación superior con prácticas centradas en el estudiante puede promoverse a pesar de una historia previa de prácticas tradicionales.

- Es posible formar comunidades virtuales para la formación continua basada en redes.

- Es posible la reconstrucción de la formación docente continua en una comunidad virtual en la educación superior.

- Los profesores pueden encontrar respuestas al proceso de cambio de su enseñanza en una comunidad de esta naturaleza. Desgraciadamente estamos en una fase experto-usuario.

La comunidad no funciona adecuadamente, y creemos que las fortalezas y debilidades pueden explicar lo que sucede y lo medios que necesitamos explorar en próximos estudios.

Los puntos fuertes fueron las siguientes:

- Los docentes participaron con gran disposición y entusiasmo en todos los procesos de formación que se presentaron. 
- Los docentes se incorporaron al proceso de formación mediante creatividad en sus propias clases.

- Este proceso promueve formas de trabajo que son útiles para el desarrollo profesional.

- La comunidad puede funcionar sin la presencia de agentes de cambio.

Las debilidades son:

- Para los profesores que no participaron, la metodología y el recurso no eran lo suficientemente atractivos, o no fueron capaces de encontrar una manera de interactuar y participar.

- Dado que los participantes necesitan a los agentes de cambio, hay que buscar alternativas para que la red funcione.

- No pasa nada que no está sucediendo en la realidad. ¿En qué medida la falta de una comunidad previa afecta el funcionamiento de la comunidad virtual? Ha habido una cierta ingenuidad con respecto a la fuerza de los temas de la creatividad y las redes sociales como una herramienta para el cambio.

- No había necesidad o demanda previa de formación, aunque la temática era demandada al ser presentada.

- Los profesores comparten un área geográfica relativamente cercana, la ciudad de México, lo que puede haber significado que la red no era necesaria y se ha sustituido por el intercambio informal. Posteriormente se nos planteó que, bien al contrario, nadie estaba interesado en cruzar la ciudad para mantener reuniones.

- El carácter voluntario de la participación era muy condicional.

- El tiempo asignado para el proyecto era demasiado corto. El cambio suele ocurrir a más largo plazo.

- Exigir pruebas de la participación inhibe la propia participación.

Queda entonces un nuevo espacio para seguir preguntándose con la propia comunidad para comprender mejor la génesis de las comunidades virtuales que procuran desarrollo profesional.

\section{Referencias bibliográficas}

FELDMAN, A. AND T. WEISS (2010) Understanding change in teachers' ways of being through collaborative action research: a cultural-historical activity. Educational action research, 18 (1), 29-55.

HERRÁN, A. DE LA (2006) Hacia una creatividad complejo-evolucionista. Redefinición del concepto de creatividad desde una educación de la conciencia. In Comprender y evaluar la creatividad. Vol. 1: Un recurso para mejorar la calidad de la enseñanza, (eds. S. de la Torre and V. Violant, 112-139). Málaga: Aljibe. 
HERRÁN, A. DE LA (2008a) Creatividad para la formación. In Compendio de Didáctica General, (ed. J.C. Sánchez Huete, 557-606). Madrid: CCS.

HERRÁN, A. DE LA (2008b) Didáctica de la creatividad. In Didáctica General: La práctica de la enseñanza en Educación Infantil, Primaria y Secundaria, (eds. A. de la Herrán y J. Paredes, 79-123). Madrid: Mc Graw-Hill.

HERRÁN, A. DE LA (2009a) Contribución al concepto de creatividad: un enfoque paquidérmico ( $1^{\mathrm{a}}$ parte). Educación y Futuro. Revista de Investigación Aplicada y Experiencias Educativas, 21, 43-70.

HERRÁN, A. DE LA (2009b) La práctica de la creatividad formativa: selección de técnicas didácticas. In La práctica de la innovación educativa, (eds. J. Paredes y A. de la Herrán, 73-104). Madrid: Síntesis.

JAMES, A. (2009) How do New Designs for Education and Education Leadership Include Concepts of "Least Intrusive Education (LIE)? Or other forms of studentdriven curriculum? Paper presented at European education research Association conference, ECER 2009, September 28.

LIEBERMAN, A. AND L. MILLER (2001) Teachers caught in the action: professional development that matters. New York: Teachers College Press.

PAREDES, J. (2011). Transformar la enseñanza universitaria con la formación mediante la creatividad. Una investigación-acción con apoyo de las tic. Revista Iberoamericana de Educación Superior (ries), vol. II, núm. 5, pp. 101-113.

PAREDES, J.; HERRÁN, A. DE LA (2011) Caceu 2010. Validación de un cuestionario. Revista ESE, 21: 41-59.

STUCKEY, B. AND S. BARAB (2007) New conceptions for community design. In The Sage handbook of e-learning research, (eds R. Andrews and C. Haythornthwaite, 439-465). Los Ángeles: SAGE.

TORRE, S. DE LA (1993) La creatividad en la aplicación del método didáctico. In Estrategias metodológicas en la formación del profesorado, (eds. M. L. Sevillano and F. Martín-Molero, 53-100). Madrid: UNED.

TORRE, S. DE LA (2006) Un modelo polivalente para evaluar la creatividad. In Comprender y evaluar la creatividad. Vol. 2: Cómo investigar y evaluar la creatividad, (eds. S. de la Torre, and V. Violant, 122-142). Málaga: Aljibe.

WENGER E. (1998). Communities of Practice: Learning, Meaning, and Identity. Cambridge: University Press.

YIN, R. (2009). Case study research. Design and methods. $4^{\mathrm{a}}$ ed. Thousand Oaks: Sage. 


\section{Correspondencia con los autores}

Joaquín Paredes-Labra

http://www.uam.es/joaquin.paredes

Universidad Autónoma de Madrid, España

Agustín de la Herrán-Gascón

http://www.uam.es/jagustin.delaherran

Universidad Autónoma de Madrid, España

Daniel Velázquez-Vázquez

Universidad Nacional Autónoma de México, México 